Abanico Veterinario. Enero-Diciembre 2021; 11:1-14. http://dx.doi.org/10.21929/abavet2021.4

Artículo Original. Recibido: 24/02/2020. Aceptado: 25/11/2020. Publicado: 04/01/2021. Clave:2020-18.

\title{
Crecimiento de corderos de pelo en el altiplano semiárido de Zacatecas durante el invierno
}

\author{
Hair lamb growth in the semiarid plateau of Zacatecas during the winter season
}

\section{López-Carlos Marco ${ }^{1 \mathrm{ID}}$, Fernández-Mier Ricardo ${ }^{1 \mathrm{ID}}$, Aréchiga-Flores Carlos ${ }^{\star 1} \mathrm{ID}$, Hernández-Briano Pedro ${ }^{1 \mathrm{ID}}$, Medina-Flores Carlos $^{1 \mathrm{ID}}$, Ramírez-Chéquer Juan ${ }^{1} \mathrm{ID}$}

1 Unidad Académica de Medicina Veterinaria y Zootecnia, Universidad Autónoma de Zacatecas, Zacatecas, México. *Autor responsable y de correspondencia: Aréchiga-Flores Carlos. Unidad Académica de Medicina Veterinaria y Zootecnia de la Universidad Autónoma de Zacatecas; Jardín Juárez No. 147, Col. Centro, Zacatecas, Zacatecas, México, CP 98000. lopcarmarco@hotmail.com, i92017@outlook.com, arechiga.uaz@gmail.com,phbriano@gmail.com, carlosmedina1@hotmail.com, cheque-r@hotmail.com.

\section{RESUMEN}

El objetivo del estudio fue determinar el efecto del sexo, tipo de parto, raza, condición de crecimiento retardado y mes de nacimiento sobre peso al nacimiento (PN), crecimiento pre- y post-destete de crías ovinas de pelo nacidas en invierno $(n=416)$. Los corderos mostraron mejor $(P<0.05)$ desempeño que las corderas. Las crías de parto sencillo pesaron más $(\mathrm{P}<0.05)$ que las de parto múltiple al nacimiento y al destete, aunque las crías de parto múltiple obtuvieron mayor $(\mathrm{P}<0.05)$ ganancia diaria de peso (GDP) postdestete. Las crías de madre Blackbelly mostraron menor $(P<0.05)$ peso y crecimiento. La progenie de sementales raza Dorper obtuvo un mayor $(P<0.05)$ peso de venta $(P V)$ y GDP post-destete que la progenie de sementales Katahdin. En las crías valoradas clínicamente con crecimiento retardado se observó un menor $(\mathrm{P}<0.05) \mathrm{PN}$ y crecimiento en general. Aunque las crías nacidas en diciembre tuvieron mayor $(\mathrm{P}<0.05) \mathrm{PN}$, las crías nacidas en febrero presentaron mayor $(\mathrm{P}<0.05)$ GDP pre-destete y $\mathrm{PV}$. En conclusión, los factores evaluados afectan significativamente tanto el PN, y el crecimiento general de las crías nacidas en época invernal en el altiplano semiárido de México.

Palabras clave: corderos, ovinos de pelo, crecimiento, retraso del crecimiento, México.

\section{ABSTRACT}

The study aimed to assess the effect of the sex, birth type, breed, growth retardation condition, and month of birth over birth weight (PN), pre-and post-weaning growth of hair-lambs that were born during winter ( $\mathrm{n}$ $=416)$. Male lambs showed better overall growth performance $(P<0.05)$ than female lambs. Single born lambs weighed more $(P<0.05)$ than multiple born lambs at birth, and weaning, although multiple born lambs had greater $(P<0.05)$ average daily gain $(G D P)$ post-weaning. Blackbelly lambs showed less weight $(P$ $<0.05)$ and growth. Dorper-sired lambs had greater $(P<0.05)$ sale weight $(P V)$ and GDP after weaning than Katahdin-sired lambs. Lambs with growth retardation had less weight $(P<0.05)$ and general performance. While lambs born in December had greater $(P<0.05) P N$, lambs born in February had greater $(P<0.05)$ pre-weaning GDP and PV. In conclusion, the evaluated factors significantly affect both PN and the overall growth performance of lambs born in winter in the semi-arid plateau of Mexico.

Keywords: lamb, hair sheep, growth, growth retardation, Mexico. 


\section{INTRODUCCIÓN}

Los ovinos tienen la capacidad de convertir forrajes toscos en proteína animal y producen con mayor eficiencia que los rumiantes mayores (San et al., 1998). Tradicionalmente, la región semiárida de México ubicada en la mesa norte del altiplano es reconocida por la producción de ganado ovino, principalmente con la raza Rambouillet en sistemas extensivos, y recientemente con razas de pelo como la Blackbelly, Pelibuey, Katahdin, Dorper y sus cruzas, en sistemas de producción semi-intensivos (Partida et al., 2013). El costo de producción en el sistema semi-intensivo es más elevado que en el sistema extensivo, debido a las inversiones necesarias en instalaciones, equipamiento, mano de obra y alimentación; así que estos sistemas, deben tener un alto nivel de productividad para que sean redituables económicamente. Por lo anterior, se debe enfatizar la adecuada selección de razas (paternas y maternas) y el manejo apropiado de los indicadores productivos desde el nacimiento hasta la venta de los corderos (Partida et al., 2013).

Las razas de ovinos de pelo, presentan una gran variabilidad en cuanto a genotipos y características productivas (Wildeus, 1997). En efecto, el genotipo y otras características afectan el desempeño reproductivo y productivo de la especie. En este sentido, las ventajas que presentan los ovinos de pelo son su baja estacionalidad reproductiva, mayor prolificidad (Galina et al., 1996), resistencia a parásitos (Vanimisetti et al., 2004) y la reducción de costos derivados de la producción y trasquila de la lana (Notter, 2000). No obstante, las razas de pelo son más pequeñas, de menor tasa de crecimiento, y masa muscular, comparado a las razas de ovinos de lana, y fueron desarrolladas para explotarse bajo condiciones de climas cálidos tropicales (Wildeus, 1997; Notter, 2000), donde las temperaturas invernales son menos frías que las prevalecientes en la región del Altiplano mexicano, en cuyos inviernos se registran masa de aire polar y temperaturas nocturnas menores a los $0^{\circ} \mathrm{C}$ (Vidal, 2005).

Sin embargo, no existen estudios en esta región que identifiquen los principales factores que influyen sobre el peso al nacer y el desarrollo de los corderos de razas de pelo en época invernal. Por lo anterior, el objetivo del presente estudio fue evaluar algunos factores como el efecto del sexo, el tipo de parto, la raza de los progenitores, la condición de retraso del crecimiento y el mes de nacimiento sobre el peso al nacimiento (PN) y crecimiento pre-destete y post-destete en corderos de pelo nacidos en época invernal en un sistema semi-intensivo de la región norte del Altiplano Mexicano.

\section{Lugar de estudio}

\section{MATERIAL Y MÉTODOS}

El presente estudio se realizó durante los meses de diciembre del año 2017 a febrero del año 2018 en el rancho San Isidro, ubicado en el municipio de Calera de Víctor Rosales, 
Zacatecas, México. La región pertenece al altiplano mexicano y se localiza geográficamente a $22^{\circ} 58^{\prime} 47^{\prime \prime}$ de latitud norte y $102^{\circ} 40^{\prime} 49^{\prime \prime}$ de longitud oeste. El clima es semiárido frío $\mathrm{BSk}$, con temperatura media anual de $17^{\circ} \mathrm{C}$, temperatura máxima media de $30^{\circ} \mathrm{C}$ en mayo, y temperatura mínima media de $3^{\circ} \mathrm{C}$ en enero, aunque se alcanzan temperaturas bajo $0^{\circ} \mathrm{C}$ durante el invierno (García, 1988).

\section{Animales y mediciones}

En el presente estudio se utilizaron 416 crías nacidas durante la época invernal (parto simple $n=242$ crías, $58.2 \%$; parto doble $n=135$ crías, $32.4 \%$; parto triple $n=39$ crías, $9.4 \%$ ). Se registró la fecha de nacimiento, el sexo (macho, hembra), la raza del padre (Dorper o Katahdin), la raza de la madre (Blackbelly, Dorper, Katahdin y Pelibuey) y el tipo de parto (sencillo, doble o triple). Además, se realizó una valoración clínica a partir de la cual se clasificó a las crías en sanos y con condición de retraso del crecimiento de acuerdo a la descripción realizada por Shelton (1964), la cual consistió en identificar a las crías con crecimiento retardado caracterizados por la presencia de pelaje hirsuto, bajo desarrollo corporal, menor vitalidad y fortaleza física; mientras que los corderos considerados como clínicamente sanos fueron aquellos que mostraron un pelaje suave, buen estado general, adecuado desarrollo corporal y vigor general. Las crías se pesaron al nacer, al destete (PD) y al momento de venta (PV) mediante báscula digital para su finalización en otras explotaciones. Los PD se ajustaron a los $75 \mathrm{~d}$ y el PV a los $145 \mathrm{~d}$ de edad. Los pesos ajustados fueron calculados como: PD a $75 \mathrm{~d}=[$ [peso al destete - peso al nacer) / edad al destete en días $] \times 75+$ peso al nacer, y PV a $145 \mathrm{~d}=[$ (peso final peso observado al destete) / días transcurridos del destete al pesaje final en días] $\times 70+$ peso al destete ajustado a $75 \mathrm{~d}$ (Thompson, 2006).

\section{Alimentación y alojamiento}

Las hembras fueron alimentadas en pastoreo diurno (pradera compuesta de rye grass anual (Lolium multiflorum Lam.), rye grass perenne (Lolium perenne), pasto bromo (Bromus willdenowii cv. Matua), festuca (Festuca arundinacea), orchard potomac (Dactylis glomerata), con encierro nocturno y suplementación a base de rastrojo de maíz, ensilado de maíz, heno de alfalfa y avena, recibiendo 300 a $350 \mathrm{~g} / \mathrm{d}$ de alimento comercial (13\% PC y $3.4 \mathrm{Mcal}$ EM/kg de MS) en el último tercio de gestación e inicio de la lactancia. Las hembras al parto fueron alojadas en corraletas techadas donde se mantuvieron junto a sus crías durante la primera semana de vida. Las crías se pesaron al nacer y se identificaron dentro de las primeras $24 \mathrm{~h}$ de vida. Posteriormente, las crías salieron a pastorear con sus madres durante cuatro a seis horas al día, con un suplemento de iniciación "creep feeding" (20\% PC y 2.9 Mcal EM/kg de MS) por la tarde. El destete se realizó a los $75 \mathrm{~d}$ (dos meses y medio de edad), y a partir de esta etapa las crías fueron engordadas con $65 \%$ de alimento comercial (16\% PC y 3.4 Mcal EM/kg de MS) y $35 \%$ de una mezcla de heno de alfalfa y avena o rastrojo molido de maíz hasta que fueron 
enviados al mercado con un peso promedio de $34.9 \pm 0.4 \mathrm{~kg}$. Los animales tuvieron libre acceso a agua fresca y a una mezcla de sales minerales en el corral.

\section{Análisis estadístico}

El análisis estadístico de los datos se realizó con el procedimiento GLM del paquete estadístico SAS (SAS Institute, Cary N.C., U.S.A.) versión 9.1. El modelo estadístico inicial incluyó los efectos principales e interacciones, aunque debido a que las interacciones entre efectos simples no fueron significativas, en el modelo reducido solo se incluyeron los efectos fijos de sexo de la cría (macho, hembra), tipo de parto (sencillo, múltiple), raza materna (Blackbelly, Dorper, Katahdin, Pelibuey), raza paterna (Dorper, Katahdin), valoración clínica (con o sin retraso del crecimiento) y mes de nacimiento (diciembre, enero, febrero). Las diferencias entre medias se establecieron mediante prueba de Tukey $(\mathrm{P}<0.05)$.

\section{RESULTADOS}

El sexo de las crías afectó $(P<0.05)$ todas las características evaluadas. Los machos fueron más pesados $(P<0.05)$ que las hembras al nacimiento (4.0 vs. $3.6 \mathrm{~kg})$, al destete $(19.0$ vs. $16.0 \mathrm{~kg}$ ) y a los $145 \mathrm{~d}$ de edad (36.0 vs. $29.0 \mathrm{~kg})$. Además, mostraron una mayor $(\mathrm{P}<0.05)$ GDP pre-destete (194 vs. $169 \mathrm{~g}$ ), GDP post-destete (246 vs. $181 \mathrm{~g})$ y GDP promedio (219 vs. $175 \mathrm{~g})$. Los machos ganaron $4.5 \mathrm{~kg}$ más peso $(\mathrm{P}<0.05)$ que las hembras entre los 75 y los $145 \mathrm{~d}$ de edad (Cuadro 1).

El tipo de parto afectó $(P<0.05)$ el crecimiento de las crías. Las crías de parto sencillo obtuvieron mayor ( $\mathrm{P}<0.05) \mathrm{PN}(4.0 \mathrm{vs} .3 .5 \mathrm{~kg})$, al destete (18 vs. $16 \mathrm{~kg})$ y a los $145 \mathrm{~d}$ de edad (32 vs. $31 \mathrm{~kg}$ ), además de una mayor ( $\mathrm{P}<0.05)$ GDP pre-destete (188 vs. $165 \mathrm{~g}$ ) y GDP promedio (195 g vs. $188 \mathrm{~g}$ ) que las crías provenientes de partos múltiples. Sin embargo, las crías de parto múltiple compensaron el menor crecimiento previo y obtuvieron una mayor ( $\mathrm{P}<0.05$ ) GDP post-destete (213 g vs. $202 \mathrm{~g}$ ), alcanzando así una mayor $(\mathrm{P}<0.05)$ ganancia de peso entre los 75 a los $145 \mathrm{~d}$ de edad (15 vs.14 kg) en comparación a las crías de parto sencillo (Cuadro 1).

La raza de la madre afectó $(\mathrm{P}<0.05)$ las características de peso corporal y GDP pre- y post-destete (Cuadro 2). En cuanto al PN, las crías de madres Pelibuey (4.0 kg) y Dorper $(3.9 \mathrm{~kg})$ fueron más pesados $(\mathrm{P}<0.05)$ en comparación con las crías de hembras Katahdin $(3.7 \mathrm{~kg})$, mientras que los corderos de madre Blackbelly obtuvieron el menor $(\mathrm{P}>0.05)$ peso $(3.0 \mathrm{~kg})$. EI PD ajustado a $75 \mathrm{~d}$ fue mayor $(\mathrm{P}<0.05)$ para los corderos de ovejas de las razas Dorper y Katahdin (18 kg y $17 \mathrm{~kg}$, respectivamente), seguido de los corderos de madre Pelibuey $(16 \mathrm{~kg}$ ) y Blackbelly $(15 \mathrm{~kg})$. Por su parte, el PV ajustado a $145 \mathrm{~d}$ fue similar $(P>0.05)$ entre los corderos de madres de raza Dorper, Katahdin y Pelibuey (32, 32 y $31 \mathrm{~kg})$, y menor $(\mathrm{P}<0.05)$ en los corderos de raza materna Blackbelly $(28 \mathrm{~kg})$. 
La GDP pre-destete $(0$ a 75 d de edad) fue mayor $(P<0.05)$ en los corderos de madres Dorper (187 g) y similar entre las razas Katahdin (171 g), Pelibuey (167 g) y Blackbelly (164 g). Sin embargo, la GDP post-destete $(75$ a $145 \mathrm{~d}$ de edad) fue mayor $(P<0.05)$ en las crías de madre Katahdin (219 g), Pelibuey (214 g) y Dorper (198 g) en relación a las crías de madres Blackbelly ( $173 \mathrm{~g}$ ). Además, la GDP promedio ( 0 a $145 \mathrm{~d}$ de edad) fue mayor $(\mathrm{P}<0.05)$ en los corderos de madre Dorper $(198 \mathrm{gr})$, Katahdin $(194 \mathrm{~g})$ y Pelibuey (185 g), en relación a las crías de madres Blackbelly (173 g).

Cuadro 1. Efecto del sexo de la cría y tipo de parto en el peso al nacimiento, al destete ajustado a $75 \mathrm{~d}$, ajustado a $145 \mathrm{~d}$, la ganancia de peso pre-destete y post-destete (media $\pm D$.E.) en corderos de pelo nacidos en invierno en un sistema semi-intensivo del Altiplano Mexicano.

\begin{tabular}{|c|c|c|c|c|}
\hline \multirow[b]{2}{*}{ Característica } & \multicolumn{2}{|c|}{ Sexo del cordero } & \multicolumn{2}{|c|}{ Tipo de parto } \\
\hline & $\begin{array}{c}\text { Macho } \\
(\mathrm{n}=160)\end{array}$ & $\begin{array}{c}\text { Hembra } \\
(\mathrm{n}=256)\end{array}$ & $\begin{array}{r}\text { Sencillo } \\
(\mathrm{n}=242)\end{array}$ & $\begin{array}{r}\text { Múltiple } \\
(\mathrm{n}=174)\end{array}$ \\
\hline \multicolumn{5}{|l|}{ Peso corporal, kg } \\
\hline Al nacimiento & $4.0 \pm 0.09^{a}$ & $3.6 \pm 0.06^{b}$ & $4.0 \pm 0.06^{a}$ & $3.5 \pm 0.08^{b}$ \\
\hline Al destete ajustado a $75 \mathrm{~d}$ & $19 \pm 0.4^{a}$ & $16 \pm 0.2^{b}$ & $18 \pm 0.4^{a}$ & $16 \pm 0.2^{b}$ \\
\hline Ajustado a $145 \mathrm{~d}$ & $36 \pm 0.6^{a}$ & $29 \pm 0.4^{b}$ & $32 \pm 0.5^{\mathrm{a}}$ & $31 \pm 0.5^{\mathrm{a}}$ \\
\hline \multicolumn{5}{|l|}{ Ganancia diaria de peso, g/d } \\
\hline Pre-destete (0 a 75 d) & $194 \pm 6^{a}$ & $169 \pm 3^{b}$ & $188 \pm 5^{\mathrm{a}}$ & $165 \pm 3^{b}$ \\
\hline Post-destete (75 a 145 d) & $246 \pm 5^{a}$ & $181 \pm 3^{b}$ & $202 \pm 4^{b}$ & $213 \pm 5^{a}$ \\
\hline Promedio (0 a $145 \mathrm{~d}$ ) & $219 \pm 4^{a}$ & $175 \pm 3^{b}$ & $195 \pm 3^{\mathrm{a}}$ & $188 \pm 3^{b}$ \\
\hline
\end{tabular}

Cuadro 2. Efecto de la raza materna y paterna en el peso al nacimiento, al destete ajustado a $75 \mathrm{~d}$, ajustado a $145 \mathrm{~d}$, la ganancia de peso pre-destete y post-destete (media $\pm D$.E.) en crías de ovinos de pelo nacidos en invierno en un sistema semi-intensivo del Altiplano Mexicano.

\begin{tabular}{|c|c|c|c|c|c|c|}
\hline \multirow[b]{2}{*}{ Característica } & \multicolumn{4}{|c|}{ Raza materna } & \multicolumn{2}{|c|}{ Raza paterna } \\
\hline & $\begin{array}{r}\text { Blackbelly } \\
(\mathrm{n}=36)\end{array}$ & $\begin{array}{r}\text { Dorper } \\
(\mathrm{n}=240)\end{array}$ & $\begin{array}{l}\text { Katahdin } \\
(\mathrm{n}=104)\end{array}$ & $\begin{array}{r}\text { Pelibuey } \\
(\mathrm{n}=36)\end{array}$ & $\begin{array}{r}\text { Dorper } \\
(\mathrm{n}=172) \\
\end{array}$ & $\begin{array}{l}\text { Katahdin } \\
(\mathrm{n}=244) \\
\end{array}$ \\
\hline \multicolumn{7}{|l|}{ Peso corporal (kg) } \\
\hline Al nacimiento & $3.0 \pm 0.13^{c}$ & $3.9 \pm 0.07^{a}$ & $3.7 \pm 0.09^{b}$ & $4.0 \pm 0.11^{a}$ & $3.8 \pm 0.08^{a}$ & $3.8 \pm 0.07^{a}$ \\
\hline Al destete ajustado a $75 \mathrm{~d}$ & $15 \pm 0.5^{b}$ & $18 \pm 0.4^{a}$ & $17 \pm 0.3^{a}$ & $16 \pm 0.3^{b}$ & $17 \pm 0.3^{a}$ & $17 \pm 0.3^{a}$ \\
\hline Ajustado a $145 \mathrm{~d}$ & $28 \pm 1.1^{\mathrm{b}}$ & $32 \pm 0.5^{\mathrm{a}}$ & $32 \pm 0.6^{a}$ & $31 \pm 0.8^{a}$ & $32 \pm 0.5^{\mathrm{a}}$ & $31 \pm 0.5^{b}$ \\
\hline \multicolumn{7}{|l|}{ Ganancia diaria de peso $(g / d)$} \\
\hline Pre-destete (0 a 75 d) & $164 \pm 6^{b}$ & $187 \pm 5^{a}$ & $171 \pm 5^{b}$ & $167 \pm 5^{b}$ & $180 \pm 4^{a}$ & $177 \pm 4^{a}$ \\
\hline Post-destete (75 a 145 d) & $182 \pm 11^{b}$ & $213 \pm 4^{a}$ & $219 \pm 6^{a}$ & $214 \pm 10^{a}$ & $212 \pm 5^{a}$ & $202 \pm 4^{b}$ \\
\hline Promedio (0 a 145 d) & $173 \pm 8^{c}$ & $198 \pm 3^{a}$ & $194 \pm 4^{a}$ & $189 \pm 6^{b}$ & $196 \pm 3^{a}$ & $189 \pm 3^{b}$ \\
\hline
\end{tabular}

La raza del padre no afectó ( $\mathrm{P}>0.05)$ el PN, el PD, o la GDP pre-destete (0-75 d de edad). Los corderos de raza paterna Dorper fueron más pesados $(\mathrm{P}<0.05)$ a los 145 de edad 
(32 kg vs. $31 \mathrm{~kg}$ ) y obtuvieron una mayor ( $\mathrm{P}<0.05)$ GDP post-destete (212 vs. $202 \mathrm{~g})$ y GDP promedio (196 vs. $190 \mathrm{~g}$ ) que los corderos de raza paterna Katahdin (Cuadro 2).

Las crías clínicamente sanas, obtuvieron un mayor $(\mathrm{P}<0.05) \mathrm{PN}$ que los corderos con retraso del crecimiento (3.9 vs. $3.4 \mathrm{~kg}$ ), dicha diferencia también se observó al destete $(18 \mathrm{vs} .13 \mathrm{~kg}$ ) y a los $145 \mathrm{~d}$ de edad (34 vs. $21 \mathrm{~kg}$ ). En el periodo post-destete (entre 75 a $145 \mathrm{~d}$ ), los corderos sanos ganaron en promedio $7 \mathrm{~kg}$ más $(\mathrm{P}<0.05)$ que los corderos con retraso del crecimiento (Cuadro 3).

Por otra parte, se observó que los corderos nacidos en el mes de diciembre $(4.6 \mathrm{~kg})$ fueron más pesados $(\mathrm{P}<0.05)$ que los nacidos en enero $(3.8 \mathrm{~kg})$, seguidos de los nacidos en febrero $(3.6 \mathrm{~kg})$. Al destete y a los $145 \mathrm{~d}$ de edad, los corderos nacidos en diciembre y enero obtuvieron pesos similares $(P<0.05)$, aunque los nacidos en febrero $(P<0.05)$ fueron $4 \mathrm{~kg}$ más pesados. Además, los corderos nacidos en febrero tuvieron mayor $(P<0.05)$ GDP pre-destete y GDP promedio. No se encontraron diferencias $(P>0.05)$ en la GDP post-destete, ni ( $P>0.05)$ en el peso ajustado a 75 y $145 \mathrm{~d}$ de edad (Cuadro 3 ).

Cuadro 3. Efecto de la condición clínica y mes de nacimiento en el peso al nacimiento, al destete ajustado a $75 \mathrm{~d}$, ajustado a $145 \mathrm{~d}$, la ganancia de peso pre-destete y post-destete (media $\pm D . E$.) en corderos de pelo nacidos en invierno en un sistema semi-intensivo del Altiplano Mexicano

\begin{tabular}{|c|c|c|c|c|c|}
\hline \multirow[b]{2}{*}{ Característica } & \multicolumn{2}{|c|}{ Valoración clínica } & \multicolumn{3}{|c|}{ Mes de nacimiento } \\
\hline & $\begin{array}{r}\text { Clínica-mente } \\
\text { sano } \\
(\mathrm{n}=332)\end{array}$ & $\begin{array}{r}\text { Retraso del } \\
\text { crecimiento } \\
(n=84) \\
\end{array}$ & $\begin{array}{c}\text { Diciembre } \\
(\mathrm{n}=20)\end{array}$ & $\begin{array}{c}\text { Enero } \\
(\mathrm{n}=316)\end{array}$ & $\begin{array}{l}\text { Febrero } \\
(n=80)\end{array}$ \\
\hline \multicolumn{6}{|l|}{ Peso corporal (kg) } \\
\hline Al nacimiento & $3.9 \pm 0.05^{a}$ & $3.4 \pm 0.13^{b}$ & $4.6 \pm 0.25^{a}$ & $3.8 \pm 0.06^{b}$ & $3.6 \pm 0.13^{c}$ \\
\hline Al destete ajustado a $75 \mathrm{~d}$ & $18 \pm 0.3^{a}$ & $13 \pm 0.2^{b}$ & $16 \pm 0.3^{b}$ & $16 \pm 0.2^{b}$ & $20 \pm 0.8^{a}$ \\
\hline Ajustado a $145 d$ & $34 \pm 0.3^{a}$ & $21 \pm 0.5^{b}$ & $30 \pm 0.7^{b}$ & $31 \pm 0.4^{b}$ & $35 \pm 0.9^{a}$ \\
\hline \multicolumn{6}{|l|}{ Ganancia diaria de peso ( $g / d$ ) } \\
\hline Pre-destete (0 a 75 d) & $192 \pm 3^{a}$ & $124 \pm 3^{b}$ & $156 \pm 4^{b}$ & $169 \pm 3^{b}$ & $223 \pm 11^{\mathrm{a}}$ \\
\hline Post-destete (75 a $145 \mathrm{~d})$ & $227 \pm 3^{a}$ & $123 \pm 5^{b}$ & $192 \pm 8^{a}$ & $206 \pm 4^{a}$ & $212 \pm 7^{a}$ \\
\hline Promedio (0 a 145 d) & $209 \pm 2^{\mathrm{a}}$ & $124 \pm 4^{b}$ & $173 \pm 5^{b}$ & $187 \pm 3^{b}$ & $218 \pm 6^{a}$ \\
\hline
\end{tabular}

\section{DISCUSIÓN}

En correspondencia con los resultados del presente trabajo, varios autores (Carrillo y Segura, 1993; Garduño et al., 2002; Vicente-Pérez et al., 2015) han reportado previamente el mayor peso y desarrollo que presentan en general los corderos sobre las corderas. Al respecto, Bores-Quintero et al. (2002) observaron que durante la etapa postdestete la GDP de los machos superó $(P<0.05)$ en $27 \%$ a la GDP de hembras, mientras que De Vargas Junior et al. (2014), Schanbacher et al. (1980) y Ghafouri-Kesbi y Notter 
(2016) concluyen que las diferencias en el peso y desarrollo entre los corderos y corderas reflejan la diferencia en el entorno endocrino y requerimientos de nutrientes entre los sexos.

Las crías nacidos en parto simple presentaron una mayor tasa de crecimiento pre-destete con respecto a aquellos de parto múltiple, lo cual coincide con lo reportado previamente por Garduño et al. (2002), Mellado, et al. (2016) y Simeonov et al., (2014). Sin embargo, los corderos de parto múltiple compensaron el menor crecimiento previo al destete y obtuvieron una mayor GDP post-destete para terminar con un peso ajustado a $145 \mathrm{~d}$ similar al de los corderos nacidos en parto sencillo. Resultados similares han sido reportados por Galaviz-Rodríguez et al. (2014), y Simeonov et al 2014, y se considera que durante el periodo pre-destete, el crecimiento está determinado en buena medida por la cantidad de leche materna, sin embargo, una vez destetados los corderos de parto múltiple, consumen más alimento y crecen a un ritmo que les permite alcanzar un peso similar a los corderos de parto simple a la edad de peso al sacrificio.

Los resultados obtenidos en el presente estudio en relación a la raza materna demuestran la importancia de la elección de los vientres que constituirán la base materna en las explotaciones ovinas, ya que de esto dependerá el peso y desarrollo de los corderos, principalmente tomando en cuenta las condiciones climáticas que se presentan en esta región donde la oscilación térmica anual presenta diferencias entre las temperaturas medias en diferentes épocas del año desde $12{ }^{\circ} \mathrm{C}$ hasta más de $20^{\circ} \mathrm{C}$ (FAO, 2020). Al respecto, Grepe (2001) menciona que el primer paso en la producción de ganado ovino comienza por la elección de la raza y el objetivo principal de la explotación.

En este sentido, la raza Dorper ha mostrado adaptación y mejor velocidad de crecimiento (210 a $330 \mathrm{~g} / \mathrm{d}$ ) bajo condiciones de pastoreo en el desierto de Sudáfrica (Cloete et al., 2000). Por su parte, la raza Katahdin desarrollada en el noreste de los Estados Unidos de América, se ha caracterizado por un buen desarrollo productivo $(236 \mathrm{~g} / \mathrm{d})$ en condiciones áridas y tropicales (Burke y Apple, 2007). Adicionalmente, López-Carlos et al. (2010) realizaron un estudio en el altiplano mexicano comparando el crecimiento postdestete en ovinos de razas de pelo, en el cual reportaron que las crías de raza Dorper $(238 \mathrm{~g} / \mathrm{d})$ obtuvieron una mayor GDP que las razas Katahdin $(226 \mathrm{~g} / \mathrm{d})$, Pelibuey $(218 \mathrm{~g} / \mathrm{d})$ y Blackbelly (188 g/d), siendo la Blackbelly la de menor desempeño productivo. Al respecto Wildeus et al. (2005) mencionan que las crías de raza Blackbelly poseen una menor GDP que los corderos de las razas Katahdin y Santa Cruz (73 g/d vs. 109 y 86 $\mathrm{g} / \mathrm{d}$, respectivamente) alimentados con dietas en base a forraje.

Los corderos de raza paterna Dorper y Katahdin presentaron PN y PD similares, en coincidencia con lo reportado por Macías-Cruz et al. (2010) quienes observaron que los cruzamientos de sementales Dorper y Katahdin con hembras Pelibuey producen crías para el abasto con tasas aceptables de crecimiento, así como buena adaptación en climas áridos. Sin embargo, la progenie de sementales Dorper obtuvieron una GDP postdestete y un PV superiores $(P<0.05)$ en comparación con los corderos de raza paterna Katahdin, lo cual coincide con lo reportado por Macías-Cruz et al. (2010) quienes refieren 
que los corderos cruzados de raza paterna Dorper obtuvieron un 16 y $25 \%$ más peso por día que los de raza paterna Katahdin o Pelibuey respectivamente. En tal sentido, se asume que las razas de mayor talla poseen una mayor velocidad de crecimiento que las razas de menor talla o peso a la madurez (Owens et al., 1993). Aunque las razas conocidas como de pelo no se han evaluado tan ampliamente como las razas productoras de lana, se ha reportado que la raza Dorper posee excelentes características de crecimiento, por lo que se recomienda su uso como raza terminal, siendo competitiva inclusive al compararla con razas cárnicas de tipo lanar (Notter et al., 2000; Snowder y Duckett, 2003).

Por otro lado, en el presente estudio se observó una frecuencia del $25 \%$ de crías que presentaron la condición de retraso del crecimiento. Lo anterior, representa el primer reporte científico de que dicha condición se presenta en ovinos en México por efecto de las bajas temperaturas invernales. Sin embargo, esta condición es de gran importancia en ganado porcino y se ha reportado como una importante causa de mortalidad y baja productividad en lechones (Wu et al., 2006). La condición de crecimiento retardado ha sido revisada por Wang et al. (2017), quienes indican que puede manifestarse en todas las especies, siendo ocasionada por una combinación de factores que comienzan desde el periodo prenatal, y se le conoce como "retraso del crecimiento intrauterino". Este problema es originado por factores genéticos, epigenéticos, de madurez materna, y ambientales como el estado nutricional de la madre, estrés, enfermedades, toxinas, etc. De acuerdo con estos autores, la restricción del crecimiento intrauterino tiene efectos negativos permanentes sobre la adaptación neonatal, la supervivencia y el crecimiento pre-destete, la eficiencia de utilización del alimento, la salud general de por vida, la composición corporal, así como el desempeño reproductivo en la vida adulta, y por lo tanto tiene importantes implicaciones en la producción animal.

En relación a las diferencias obtenidas en el peso de los corderos en el invierno, se ha demostrado que las ovejas preñadas expuestas a temperaturas ambientales bajas sufren adaptaciones metabólicas para satisfacer el aumento del gasto energético asociado con el frío, lo que conducen a movilizar grasa corporal y liberar glucosa por el hígado, la cual cruza la placenta y aumenta el suministro de glucosa al feto, estimulando la secreción de insulina y el crecimiento fetal sin riesgo aparente de cetosis clínica (Kenyon et al., 2006; Norouzian, 2015; Symonds et al., 1986; Thompson et al., 1982). Recientemente, Piquer et al. (2017), explican que la exposición de ratas preñadas a $4^{\circ} \mathrm{C}$ por $3 \mathrm{~h}$ diarias causó un aumento de los niveles de norepinefrina y corticosterona en la circulación materna, y la disminución de la capacidad placentaria para eliminar la norepinefrina del feto hacia la circulación de la madre, lo que ocasionó un aumento del peso de la placenta y del peso corporal de las crías. Las temperaturas ambientales bajo las cuales se desarrolló el presente estudio fueron más frías en diciembre (media de $10.5^{\circ} \mathrm{C}$ y mínima de $-9^{\circ} \mathrm{C}$ ) que en enero (media de $11.6^{\circ} \mathrm{C}$ y mínima de $-2^{\circ} \mathrm{C}$ ) y febrero (media de $13.9^{\circ} \mathrm{C}$ y mínima de - 
$2^{\circ} \mathrm{C}$ ), lo cual probablemente explique por qué se obtuvieron pesos al nacimiento mayores en los corderos nacidos en el mes de diciembre.

Las condiciones ambientales adversas debido al efecto combinado de baja temperatura, precipitación y viento en la época de parición son la principal causa de mortalidad en corderos de pelo por el síndrome de inanición-exposición (Refshauge et al., 2016). Además, Mellor y Stafford (2004), mencionan que los ovinos de pelo muestran mayor susceptibilidad al enfriamiento que los ovinos de lana, haciéndolos más susceptibles a enfermedades respiratorias bajo condiciones de frío y humedad. En este sentido, Saravia y Cruz (2003) mencionan que, al bajar la temperatura del aire por debajo de la temperatura crítica mínima $\left(10^{\circ} \mathrm{C}\right)$ se llega al punto donde la producción de calor metabólico es insuficiente para mantener la temperatura del cuerpo y conseguir ganancias de peso en corderos. Lo anteriormente expuesto explicaría por qué los corderos nacidos en el mes de diciembre, a pesar de haber obtenido un mayor PN, posteriormente observaron una menor ganancia de peso que los corderos nacidos en enero y febrero.

Es importante considerar la raza materna, la raza paterna y el mes de nacimiento en la planeación y manejo de las explotaciones semi-intensivas con ovinos de pelo, particularmente en las condiciones climáticas y de producción de la zona norte del Altiplano Mexicano.

\section{CONCLUSIÓN}

Las crías ovinas nacidas en partos sencillos y múltiples a pesar de mostrar diferente PN, alcanzan un peso similar después del destete, y la raza de la madre determina el PN y el crecimiento de los corderos, siendo poco recomendable programar partos de hembras Blackbelly en los meses de invierno. La raza del semental (Dorper o Katahdin) no afecta el peso al nacer ni el crecimiento inicial del cordero, aunque determina el crecimiento post-destete, por lo que es recomendable el uso de la raza Dorper para mejorar el peso de venta a los $145 \mathrm{~d}$ de edad. Los corderos con retraso del crecimiento poseen un menor desempeño productivo que los corderos que no presentan ese retraso. Por lo que es recomendable reducir al máximo la incidencia de corderos con retraso del crecimiento. Los corderos nacidos en el mes de diciembre poseen un mayor peso al nacer, aunque posteriormente obtienen ganancias de peso inferiores a las obtenidas por los corderos nacidos en los meses de enero o febrero.

\section{IMPLICACIÓN}

Es importante considerar la raza materna, la raza paterna y el mes de nacimiento en la planeación y manejo de las explotaciones semi-intensivas con ovinos de pelo, particularmente en las condiciones climáticas y de producción de la zona norte del Altiplano Mexicano. Además, proporcionar mejores condiciones nutricionales a las hembras durante la gestación, parto y lactancia, para favorecer un mejor desarrollo de corderos y evitar la condición de retraso del crecimiento, ya que estos factores afectan 
de manera importante el peso al nacer y el desarrollo de los corderos antes y después del destete.

\section{LITERATURA CITADA}

BORES-QUINTERO RF, Velázquez Madrazo PA, Heredia, Aguilar M. 2002. Evaluación de razas terminales en esquemas de cruza comercial con ovejas de pelo $\mathrm{F} 1$. Técnica Pecuaria en México. 40(1): 71-79. ISSN: 0040-1889.

htpps://www.redalyc.org/articulo.oa?id=613/61340104

BURKE JM, Apple JK. 2007. Growth performance and carcass traits of forage-fed hair sheep wethers. Small Ruminant Research. 67(2-3): 264-270. ISSN: 0921-4488. https://doi.org/10.1016/j.smallrumres.2005.10.014

CARRILLO L, Segura JC. 1993. Environmental and genetic effects on preweaning growth performance of hair sheep in México. Tropical Animal Health and Production. 25(3): 173178. ISSN: 1573-7438. https://doi.org/10.1007/BF02236237

CLOETE SWP, Snyman MA, Herselman MJ. 2000. Productive performance of Dorper sheep. Small Ruminant Research.36(2): 119-135. ISSN: 0921-4488. https://doi.org/10.1016/S0921-4488(99)00156-X

DE VARGAS JUNIOR FM, Martins CF, dos Santos Pinto G, Ferreira MB, de Almeida Ricardo H, Leão AG, Mendes Fernandes AR, Teixeira A. 2014. The effect of sex and genotype on growth performance, feed efficiency, and carcass traits of local sheep group Pantaneiro and Texel or Santa Inês crossbred finished on feedlot. Tropical Animal Health and Production. 46(5):869-875. ISSN: 0049-4747. https://doi.org/10.1007/s11250-0140579-4

FAO. 2020. Estado de la diversidad biológica de los árboles y bosques en el Norte de México. $1 . \quad$ Características ecológicas y socio-económicas. http://www.fao.org/3/j0529s/j0529s01.htm\#P84_5714

GALAVIZ-RODRÍGUEZ JR, Ramírez-Bribiesca JE, Vargas-López S, Zaragoza-Ramírez JL, Guerrero-Rodríguez JD, Mellado-Bosque M, Ramírez RG. 2014. Effect of three production systems of central Mexico on growth performance of five lamb genotypes. Journal of Animal and Plant Sciences. 24(5): 1303-1308. ISSN: 1018-7081. http://www.thejaps.org.pk/docs/v-24-5/05.pdf

GALINA MA, Morales R, Silva E, López B. 1996. Reproductive performance of Pelibuey and Blackbelly sheep under tropical management systems in Mexico. Small Ruminant Research. 22(1): 31-37. https://doi.org/10.1016/0921-4488(95)00878-0 
GARCÍA E. 1988. Modificaciones al sistema de clasificación climática de Köppen. Instituto de Geografía, UNAM. Offset Larios. México, D.F. ISBN: 970-32-1010-4 http://www.publicaciones.igg.unam.mx/index.php/ig/catalog/view/83/82/251-1

GARDUÑO RG, Hernández GT, Álvarez MC. 2002. Crecimiento de corderos Blackbelly entre el nacimiento y el peso final en el trópico húmedo de México. Veterinaria México. 33(4): 443-453. ISSN: 0301-5092. https://www.redalyc. org/articulo.oa?id=42333408

GHAFOURI-KESBI F, Notter. 2016. Sex influence on genetic expressions of early growth in Afshari lambs. Archives Animal Breeding. 59(1): 9-17. ISSN: 0003-9438. https://doi.org/10.5194/aab-59-9-2016

GREPE N. 2001. Crianza de Ovinos. Centro de Estudios Agropecuarios. Editorial Iberoamericana S.A. de C.V. ISBN:970-62-5264-9.

htpp://www.icamex.edomex.gob.mx/ovinos

KENYON PR, Revell DK, Morris ST. 2006. Mid-pregnancy shearing can increase birthweight and survival to weaning of multiple-born lambs under commercial conditions. Australian Journal of Experimental Agriculture. 46(7): 821-825. ISSN: 14465574. https://doi:10.1071/EA05329

LÓPEZ-CARLOS MA, Ramírez RG, Aguilera-Soto JI, Aréchiga CF, Rodríguez H. 2010. Size and shape analyses in hair sheep ram lambs and its relationships with growth performance. Livestock Science. 131(2-3): 203-211. ISSN: 1871-1413. https://doi.org/10.1016/j.livsci.2010.04.001

MACÍAS-CRUZ U, Álvarez-Valenzuela FD, Rodríguez-García J, Correa-Calderón A, Torrentera-Olivera NG, Molina-Ramírez L, Avendaño-Reyes L. 2010. Growth and carcass traits in pure Pelibuey lambs and crosses F1 with Dorper and Katahdin breeds in confinement. Archivos de Medicina Veterinaria. 42(3): 147-154. ISSN: 0301-732X. https://www.cabdirect.org/cabdirect/search/?q=sn\%3a\%220301-732X\%22

MELLADO M, Macías U, Avendaño L, Mellado J, García E. 2016. Growth and preweaning mortality of Katahdin lamb crosses. Revista Colombiana de Ciencias Pecuarias. 29(4): 288-295. ISSN: 0120-0690. https://dx.doi.org/10.17533/udea.rccp.v29n4a06

MELLOR DJ, Stafford KJ. 2004. Animal welfare implications of neonatal mortality and morbidity in farms animals. The Veterinary Journal. 168(2): 118-133. ISSN: 10900233. https://dx.doi.org/10.1016/j.tvjl.2003.08.004 
NOROUZIAN MA. 2015. Effects of lambing season, birth type and sex on early performance of lambs. New Zealand Journal of Agricultural Research. 58(1): 84-88. ISSN: 0028-8233. https://doi.org/10.1080/00288233.2014.944270

NOTTER DR. 2000. Potential for hair sheep in the United States. Journal of Animal Science. $\quad$ 77(suppl_E): 1-8. ISSN: 1525-3163. https://doi.org/10.2527/jas2000.77ESuppl1h

OWENS FN, Dubeski P, Hanson CF. 1993. Factors that alter the growth and development of ruminants. Journal of Animal Science. 71(11): 3138-3150. ISSN: 1525-3163. https://doi.org/10.2527/1993.71113138x

PARTIDA JA, Braña-Varela D, Jiménez-Severiano H, Ríos-Rincón FG, RodríguezGermán B. 2013. Manual de Producción de Carne Ovina. Libro Técnico No. 5. Centro Nacional de Investigación Disciplinaria en Fisiología y Mejoramiento Animal. Instituto Nacional de Investigaciones Forestales, Agrícolas y Pecuarias, México. ISBN: 978-60737-0036-8. https://www.coursehero.com/file/48139668/Manual-Producci\%C3\% B3n- deCarne-Ovinapdf

PIQUER B, Fonseca JL, Lara HE. 2017. Gestational stress, placental norepinephrine transporter and offspring fertility. Reproduction. 153(2): 147-155. ISSN: 1741-7899. https://doi.org/10.1530/REP-16-0312

REFSHAUGE G, Brien FD, Hinch GN, van de Ven R. 2016. Neonatal lamb mortality: factors associated with the death of Australian lambs. Animal Production Science. 56(4): 726-735. ISSN : 1836-5787. http://dx.doi.org/10.1071/AN15121

SAN C, Sanchez A, Alfonso M. 1998. Small ruminant production systems and factors affecting lamb meat quality. Meat Science. 49: S29-S64. https://doi.org/10.1016/S03091740(98)90037-7

SARAVIA C, Cruz C. 2003. Influencia del ambiente atmosférico en la adaptación y producción animal. Montevideo. Universidad de la República Facultad de Agronomía, Nota Técnica 50. Universidad de la República de Uruguay, Montevideo, Uruguay. http://dt.csic.edu.uy/adjuntos/produccion/662_academicas_academicaarchivo.pdf

SCHANBACHER BD, Crouse JD, Ferrell CL. 1980. Testosterone influences on growth, performance, carcass characteristics and composition of young market lambs. Journal of Animal Science. 51(3): 685-691. ISSN: 1525-3163. https://digitalcommons.unl.edu/usdaarsfacpub/760 
SHELTON M. 1964. Relation of environmental temperature during gestation to birth weight and mortality of lambs. Journal of Animal Science. 23(2): 360-364. ISSN: 15253163. https://doi.org/10.2527/jas1964.232360x

SIMEONOV M, Todorov N, Nedelkov KA, Kirilov A, Harmon DL. 2014. Influence of live weight, sex and type of birth on growth and slaughter characteristics in early weaned lambs. Small Ruminant Research. 121(2-3): 188-192. ISSN: 0921-4488. https://doi.org/10.1016/j.smallrumres.2014.09.005

SNOWDER GD, Duckett SK. 2003. Evaluation of the South African Dorper as a terminal sire breed for growth, carcass, and palatability characteristics. Journal of Animal Science. 81(2): 368-375. ISSN: 1525-3163. https://doi.org/10.2527/2003.812368x

SYMONDS ME, Bryant MJ, Lomax MA. 1986. The effect of shearing on the energy metabolism of the pregnant ewe. British Journal of Nutrition. 56(3): 635-643. ISSN: 14752662. https://doi.org/10.1079/BJN19860144

THOMPSON GE, Bassett JM, Samson DE, Slee J. 1982. The effects of cold exposure of pregnant sheep on foetal plasma nutrients, hormones and birth weight. British Journal of Nutrition. 48(1): 59-64. ISSN: 1475-2662. https://doi.org/10.1079/bjn19820087

THOMPSON JM. 2006. Sheep Production Guide, EM 8916-E. Oregon State University. https://catalog.extension.oregonstate.edu/em8916

VANIMISETTI HB, Greiner SP, Zajac AM, Notter DR. 2004. Performance of hair sheep composite breeds: resistance of lambs to Haemonchus contortus. Journal of Animal Science. 82(2): 595-604. ISSN: 1525-3163. https://doi.org/10.2527/2004.822595x

VICENTE-PÉREZ R, Avendaño-Reyes L, Álvarez FD, Correa-Calderón A, Meza-Herrera CA, Mellado M, Quintero JA, Macías-Cruz U. 2015. Comportamiento productivo, consumo de nutrientes y productividad al parto de ovejas de pelo suplementadas con energía en el preparto durante verano e invierno. Archivos de Medicina Veterinaria. 47(3): 301-309. ISSN 0301-732X. http://dx.doi.org/10.4067/S0301-732X2015000300006

VIDAL ZR. 2005. Las regiones climáticas de México. Instituto de Geografía, Universidad Nacional Autónoma de México (UNAM), México, D.F. Pp. 76. ISBN: 970-32-2394-X. https://biblat.unam.mx/es/revista/investigaciones-geograficas-instituto-de-geografiaunam/2 
WILDEUS S. 1997. Hair sheep genetic resources and their contribution to diversified small ruminant production in the United States. Journal of Animal Science. 75(3): 630-640. ISSN: 1525-3163. https://doi.org/10.2527/1997.753630x

WILDEUS S, Turner KE, Collins JR. 2005. Growth Performance of Barbados Blackbelly, Katahdin, and St. Croix Hair Sheep Lambs Fed Pasture- or Hay-based Diets. Sheep and Goat Research Journal. 20:37-41. ISSN: 1535-2587. https://digitalcommons.unl.edu/usdaarsfacpub/458?utm_source=digitalcommons.unl.ed u\%2Fusdaarsfacpub\%2F458\&utm_medium=PDF\&utm_campaign=PDFCoverPages

WANG J, Feng C, Liu T, Shi M, Wu G, Bazer FW. 2017. Physiological alterations associated with intrauterine growth restriction in fetal pigs: causes and insights for nutritional optimization. Mol. Reprod. Dev. 84:897-904. ISSN: 1098-2795. https://doi.org/10.1002/mrd.22842

WU G, Bazer FW, Wallace JM, Spencer TE. 2006. Board-invited review: intrauterine growth retardation: implications for the animal sciences. Journal of Animal Sience. 84(9): 2316-2337. ISSN: 1525-3163. https://doi.org/10.2527/jas.2006-156 